\title{
A Broadband and Scalable On-Chip Inductor Model Appropriate for Operation Modes of Varying Substrate Resistivities
}

\author{
Jyh-Chyurn Guo, Senior Member, IEEE, and Teng-Yang Tan
}

\begin{abstract}
A broadband and scalable model is developed to accurately simulate on-chip inductors with various dimensions and substrate resistivities. The broadband accuracy is proven over frequencies of up to $20 \mathrm{GHz}$, even beyond resonance. A new scheme of resistance-inductance-capacitance networks is deployed for the spiral coils and substrate to account for 3-D eddy current, substrate return path, and spiral coil to substrate coupling effects. The 3-D eddy current is identified as the key element essential to accurately simulate the broadband characteristics. Electromagnetic simulation using the Advanced Design System momentum is conducted to predict the on-chip inductor performance corresponding to a wide range of substrate resistivities $\left(\rho_{\mathrm{Si}}=0.05-1 \mathrm{k} \Omega \cdot \mathrm{cm}\right)$. Three operation modes such as transverse electromagnetic mode, slow-wave mode, and eddy current mode are reproduced. The model parameters manifest themselves as physics-based through relevant correlation with $\rho_{\mathrm{Si}}$ over three operation modes. The onset of slow-wave mode can be consistently explained by a key element $R_{P}$ introduced in our model, which accounts for the conductor loss due to an eddy current arising from magnetic field coupling through a substrate return path. This broadband and scalable model is useful for radio frequency circuit simulation. In addition, it can facilitate an optimum design of on-chip inductors through the physics-based model parameters relevant to varying substrate resistivities.
\end{abstract}

Index Terms-Broadband, eddy current, inductor, scalable, substrate resistivity.

\section{INTRODUCTION}

$\mathbf{T}$ HE ON-CHIP inductor model is one of the most challenging topics for Si-based RF IC design due to difficulties in broadband accuracy, scalability, and physical interpretation of the complicated electromagnetic (EM) coupling through a semiconducting substrate by lumped elements. Many publications reported an improvement on the commonly adopted $\pi$ model [1], [2]. However, a limited bandwidth to a few gigahertz remains an open issue. A two- $\pi$ model was proposed to improve the accuracy beyond self-resonance frequency $f_{\mathrm{SR}}$ [3]. Unfortunately, this two- $\pi$ model suffers a singular point above $f_{\mathrm{SR}}$ [4]. Furthermore, the complicated circuit topology

Manuscript received January 16, 2007; revised May 17, 2007. This work was supported in part by the National Science Council under Grant NSC 95-2221E009-289 and Grant NSC 96-2221-E009-186. The review of this paper was arranged by Editor H. S. Momose.

The authors are with the Department of Electronics Engineering, National Chiao Tung University, Hsinchu 30010, Taiwan, R.O.C. (e-mail: jcguo@mail. nctu.edu.tw).

Color versions of one or more of the figures in this paper are available online at http//ieeexplore.ieee.org.

Digital Object Identifier 10.1109/TED.2007.907186 with double element number will add difficulty in parameter extraction and greater time consumption in circuit simulation [5]. In our previous work, a T-model of a new circuit scheme was created to realize three primary features, i.e., broadband accuracy, scalability, and parameter extraction flow capable of automation by Agilent IC-cap or other extraction tools [6]. However, there remained two major points deserving deeper study for enhancement. One is the precise matching of broadband $S$-parameters beyond resonance, and another is the prediction of broadband features associated with varying substrate resistivities. In this paper, an improved T-model is devised to enhance the broadband accuracy beyond resonance. The earlier resonance suffered by larger spiral coils generally reveals lower $f_{\mathrm{SR}}$, maybe far below $20 \mathrm{GHz}$. It brings a challenge to most of the lump element models and even to EM simulators to achieve precise matching with measurement beyond resonance. In our study through 3-D EM simulation [Ansoft High Frequency Structure Simulation (HFSS)], we identify that the substrate eddy current effect is actually a 3-D coupling behavior rather than a simplified planar feature. The 3-D eddy current effect plays a major role in frequency dependence beyond resonance for the on-chip inductors. In this improved T-model, three branches of parallel $R L$ elements are deployed to emulate the 3-D eddy current effect. All the equivalent circuit elements are kept constant independent of frequencies and can be expressed by a closed form derived from circuit analysis. With regard to substrate resistivity effect on inductors' frequency dependence and performance, EM simulation using Advanced Design System (ADS) momentum with an extensive calibration is conducted to accurately predict the broadband characteristics under a wide range of substrate resistivities $\left(\rho_{\mathrm{Si}}=0.05-1 \mathrm{k} \Omega\right.$. $\mathrm{cm}$ ). Three operation modes such as transverse electromagnetic (TEM) mode, slow-wave mode, and eddy current mode [7] are reproduced. The T-model parameters manifest themselves as physics-based through the relevant correlation with $\rho_{\mathrm{Si}}$ over three operation modes. It is expected that this scalable inductor model can effectively improve RF circuit simulation accuracy over broadband and facilitate the design optimization using onchip inductors.

\section{TECHNOLOGY AND CHARACTERIZATION}

Spiral inductors with square coils were fabricated by $0.13-\mu \mathrm{m}$ back-end of line (BEOL) technology with eight layers of $\mathrm{Cu}$ and fluorinated silicated glass intermetal dielectric (FSG IMD) $(k=3.8)$. The top metal, which is a 


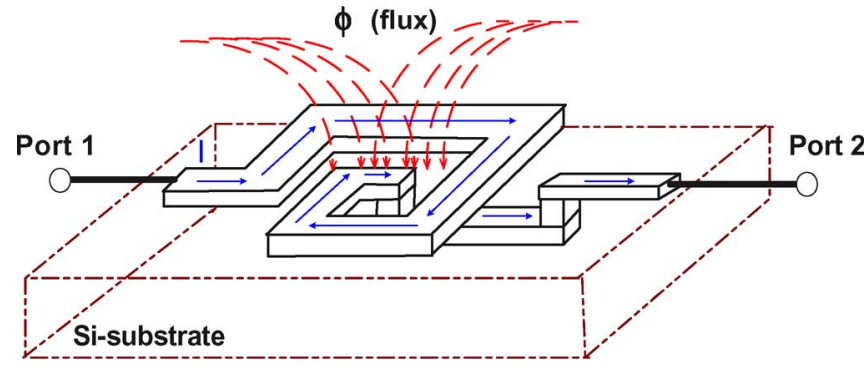

(a)

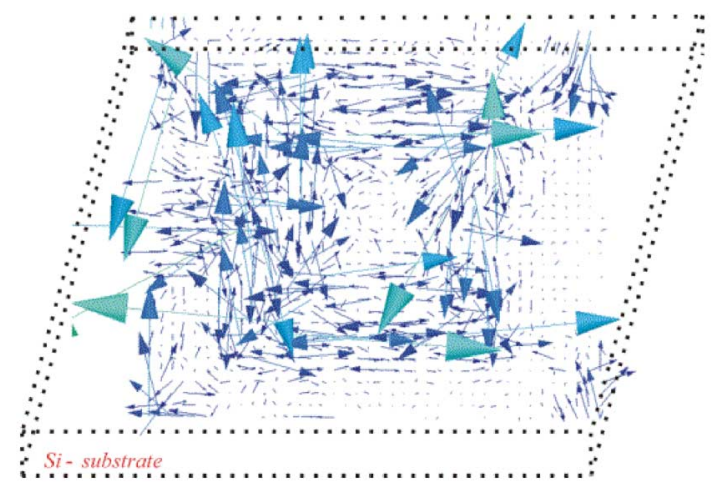

(b)

Fig. 1. (a) Three-dimensional structure of the spiral inductor that was employed for HFSS in which the current flow on the coils and the magnetic flux through the coil center are illustrated. (b) Simulated eddy current on the Si substrate in which the 3-D distribution is obviously revealed by the vectors' directions.

3- $\mu \mathrm{m} \mathrm{Cu}$, was used to implement the spiral coils to minimize series resistance. Two categories of inductors were designed with various geometries for performance benchmark and scalability verification. The first category is with a fixed metal trace width but with varying coil numbers. The metal width is fixed at $15 \mu \mathrm{m}$ and the intercoil space at $2 \mu \mathrm{m}$. The inner radius is $60 \mu \mathrm{m}$, and the outer radius is determined by different coil numbers, i.e., $N=2.5,3.5,4.5,5.5$. The physical inductance that was achieved at sufficiently low frequency is around $1.96-8.66 \mathrm{nH}$, corresponding to $N=2.5-5.5$. The second one is with a fixed coil number $N$ of 1.5 but with varying metal trace widths $W$ of $3,9,15$, and $30 \mu \mathrm{m}$. The inner radius is also $60 \mu \mathrm{m}$, and the outer radius is determined by different widths. The inductances associated with the aforementioned width splits are around $0.8-0.9 \mathrm{nH}$. The $S$-parameters were measured by using Agilent network analyzer up to $20 \mathrm{GHz}$. A two-step deembedding involving open and through dummy pads was carefully performed to yield the intrinsic characteristics for model parameter extraction.

\section{IMPROVED T-MODEL's EQUIVALENT CIRCUIT AND MOdEl PARAMETER EXTRACTION FLOW}

A 3-D EM simulation using Ansoft HFSS was performed in this paper to facilitate the understanding of EM field transport and coupling effect for spiral inductors on a semiconducting substrate such as bulk Si. Fig. 1(a) depicts the 3-D structure of the spiral inductor employed for HFSS in which the current flow on the coils and the magnetic flux through the coil center are illustrated. Fig. 1(b) presents the simulated eddy currents on the

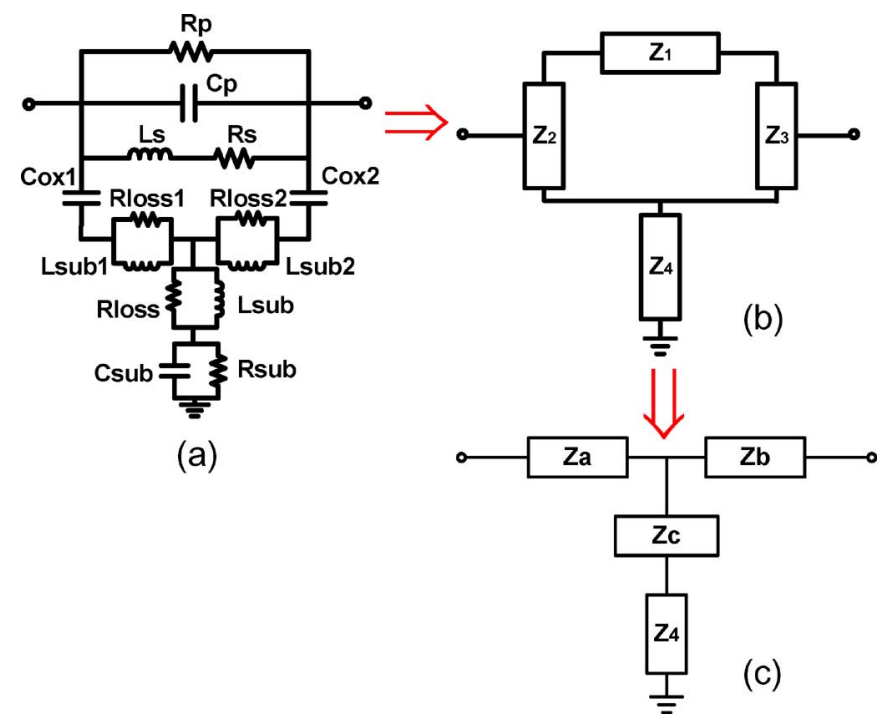

Fig. 2. Improved T-model. (a) Equivalent circuit schematics. (b) and (c) schematic block diagram for circuit analysis.

Si substrate wherein the 3-D distribution is obviously revealed by the vectors' directions. Through the 3-D EM simulation, an important insight that substrate eddy current effect is actually a 3-D coupling behavior rather than a simplified planar feature is inferred, and an improved equivalent circuit model is initiated to obtain the desired broadband accuracy.

Fig. 2(a) illustrates the circuit schematics of the improved T-model that was proposed to realize better broadband accuracy for on-chip inductors. The major enhancement over our original work [6] is the deployment of three branches of parallel $R L$ elements using a T-shape configuration to emulate the 3-D eddy current effect. Two branches of parallel $R L$ in series with $C_{\mathrm{ox} 1} / C_{\mathrm{ox} 2}$ account for the eddy current component that is normal to the substrate plane, and the other $R L$ element in series with substrate resistance-capacitance represents the eddy currents in the plane. The 3-D eddy current effect plays a major role in frequency dependence beyond resonance for the onchip inductors. Note that the pair of parallel $R L$ in series with $C_{\mathrm{ox} 1} / C_{\mathrm{ox} 2}$ is introduced for simulating the normal components of the substrate eddy current. The proposed T-shape parallel $R L$ network can enable a much better match with broadband characteristics in very high frequencies beyond $f_{\mathrm{SR}}$ (refer to Figs. 4 and 5 in Section IV).

In this way, two resistance-inductance-capacitance $(R L C)$ networks with four physical elements each are linked through $C_{\mathrm{ox} 1,2}$ in series with parallel $R L$ to account for the EM coupling between the spiral inductors and the lossy substrate underneath. Fig. 2(b) indicates the block diagrams that were derived by circuit analysis theory to extract the physical circuit elements as proposed. $Z_{1}$ represents the $R L C$ network for spiral inductor, and $Z_{4}$ is another one representing the lossy substrate. $Z_{2}$ and $Z_{3}$ consisting of $C_{\mathrm{ox} 1,2}$ and parallel $R L$ as proposed for the normal component of eddy currents act as the coupling path between $Z_{1}$ and $Z_{4}$. The circuit scheme is further transformed to Fig. 2(c) to correlate with $Y$-parameters from a two-port measurement.

As a result, all the physical elements composing the model can be extracted by the flow that is shown in Fig. 3. The 


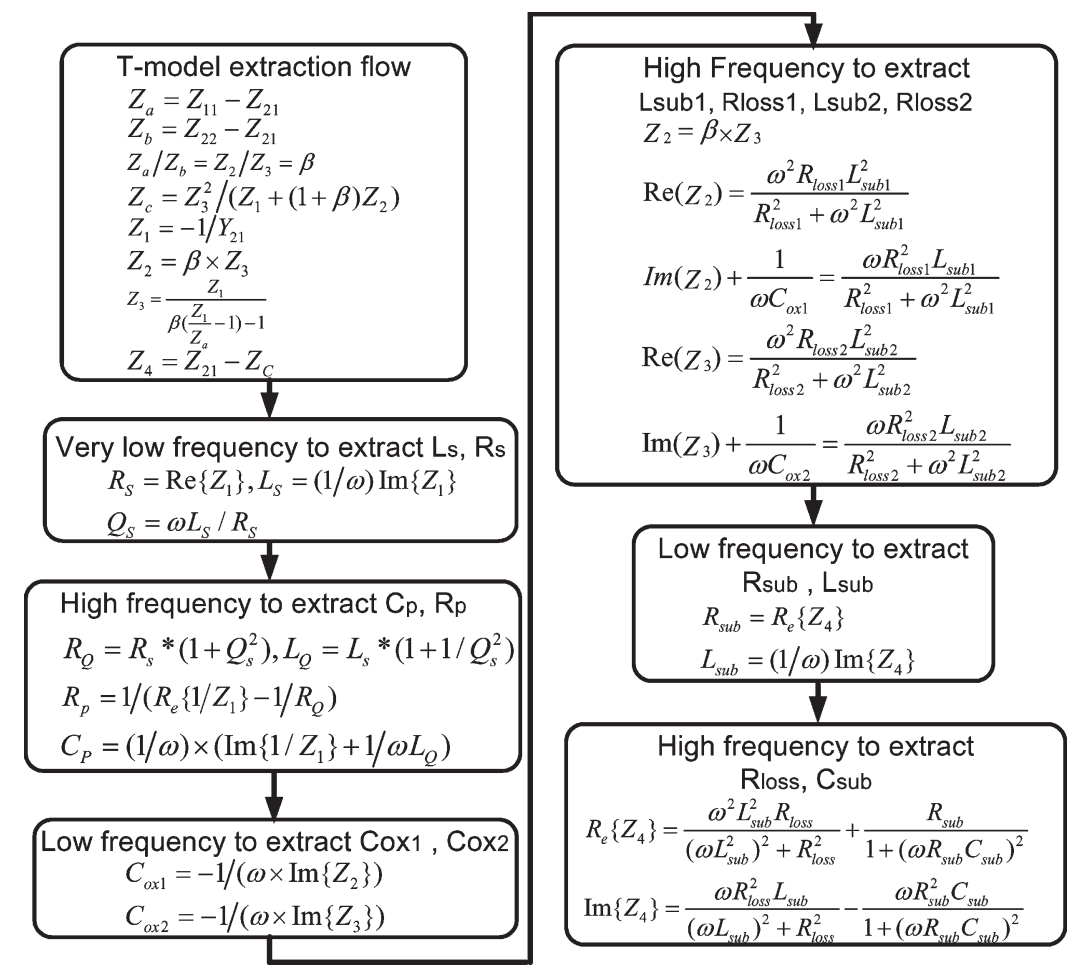

Fig. 3. Improved T-model parameter formulas and model parameter extraction flow.

details of extraction for the circuit elements in two primary $R L C$ networks, i.e., $Z_{1}$ and $Z_{4}$, and the physical properties were discussed in our previous work [6]. It is noted that $R_{S}$ and $L_{S}$ are extracted under very low frequencies when the parallel $R_{P} C_{P}$ is nearly an open circuit compared with the series $R_{S} L_{S}$. After the extraction of $R_{S}$ and $L_{S}, R_{P}$ and $C_{P}$ can be extracted in relatively higher frequencies with an appropriate range of $0.5 f_{\mathrm{SR}}-f_{\mathrm{SR}}$, where the conduction through the parallel $R_{P} C_{P}$ can no longer be neglected. With regard to the extraction of new elements added in this improved T-model, i.e., $L_{\text {sub1 } 1 /} / R_{\text {loss1 }}$ and $L_{\text {sub2 } 2} / / R_{\text {loss2 }}$, the first step is to extract $C_{\mathrm{ox} 1}$ and $C_{\mathrm{ox} 2}$ from $Z_{2}$ and $Z_{3}$ under a very low frequency provided that $L_{\mathrm{sub} 1} / / R_{\text {loss } 1}$ and $L_{\mathrm{sub} 2} / / R_{\text {loss2 }}$ are negligibly small impedances compared to $1 / \omega C_{\mathrm{ox} 1}$ and $1 / \omega C_{\mathrm{ox} 2}$, respectively. After the extraction of $C_{\mathrm{ox} 1}$ and $C_{\mathrm{ox} 2}$, the four new elements $\left(L_{\mathrm{sub} 1}, R_{\mathrm{loss} 1}\right)$ and $\left(L_{\mathrm{sub} 2}, R_{\mathrm{loss} 2}\right)$ can be derived easily from $Z_{2}$ and $Z_{3}$ under very high frequencies.

Note that $R_{P}$ is a critical element introduced in our model to simulate the substrate and conductor loss, as well as $Q$ degradation in higher frequencies prior to resonance [6]. The physical mechanism underlying the excess energy loss and $Q$ degradation and the role played by $R_{P}$ is proposed as follows. Initially, the current flow in the spiral coils builds a magnetic field, i.e., the primary magnetic flux. This primary magnetic flux will induce an eddy current in the semiconducting substrate according to Faraday's law. The generated substrate eddy current, following Lenz's law, is with a direction opposite to the original one in the spiral coils. Then, a secondary magnetic flux with an opposite direction to the primary one can be introduced by the substrate eddy current according to Ampere's law. In addition, following Faraday's law, this sec- ondary magnetic flux will further create an eddy current in the spiral coil and influence the coil current distribution. The aforementioned process that is governed by the fundamental EM field theory is called a "substrate return path." The aggravated nonuniform current distribution will introduce an excess conductor loss in the metal coils that is considered as a skin effect loss and lead to further $Q$ degradation. This mechanism explains why the new parameter $R_{P}$ that is introduced in our model is an essential element to simulate the broadband characteristics for on-Si-chip inductors.

\section{BROADBAND ACCURACY AND SCALABILITY}

The improved T-model has been extensively verified by comparison with the measurement in terms of $S$-parameters ( $S_{11}$ and $\left.S_{21}\right), L(\omega), \operatorname{Re}\left(\mathrm{Z}_{\text {in }}(\omega)\right)$, and $Q(\omega)$ over a wideband of up to $20 \mathrm{GHz}$. The scalability is rigorously evaluated over all model parameters for two categories of geometry splits, i.e., one with various coil numbers $(N=2.5,3.5,4.5,5.5)$ at a fixed width and the other with varying widths $(W=3,9,15,30 \mu \mathrm{m})$ under a fixed coil number.

\section{A. Broadband Accuracy for Various Geometries}

The broadband accuracy is justified by a good agreement with measurement in terms of the aforementioned performance parameters. Fig. 4(a)-(d) indicates the comparison between the improved T-model, original T-model, and measurement for the magnitude and phase of $S_{21}$. An excellent match is achieved for all coil numbers even beyond resonance, which happened at $f_{\mathrm{SR}} \ll 20 \mathrm{GHz}$ for larger coil numbers $(N=3.5,4.5,5.5)$. It is an obvious improvement over the original $\mathrm{T}$-model, 

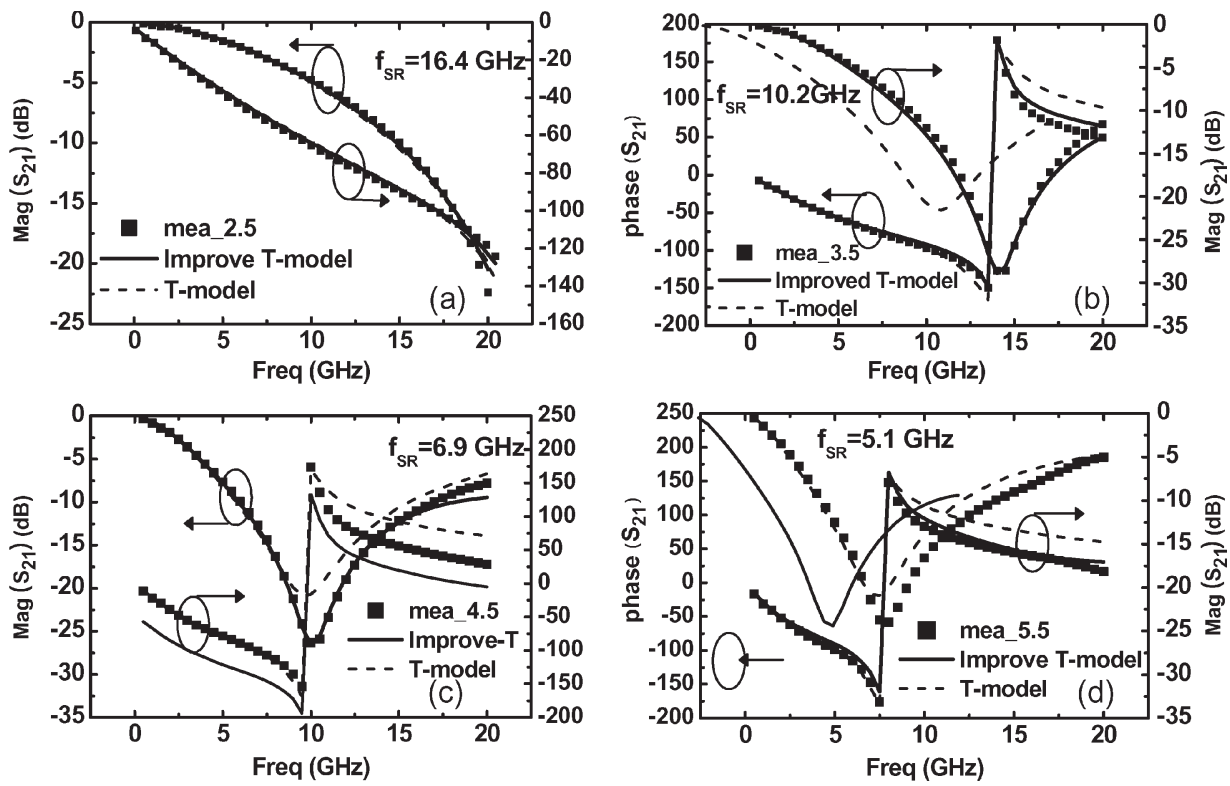

Fig. 4. Comparison between the improved T-model, original T-model, and measurement for $S_{21}$ (magnitude, phase) of inductors with various coil numbers. (a) $N=2.5$. (b) $N=3.5$. (c) $N=4.5$. (d) $N=5.5$. Improved T-model (solid lines). Original T-model (dash lines).
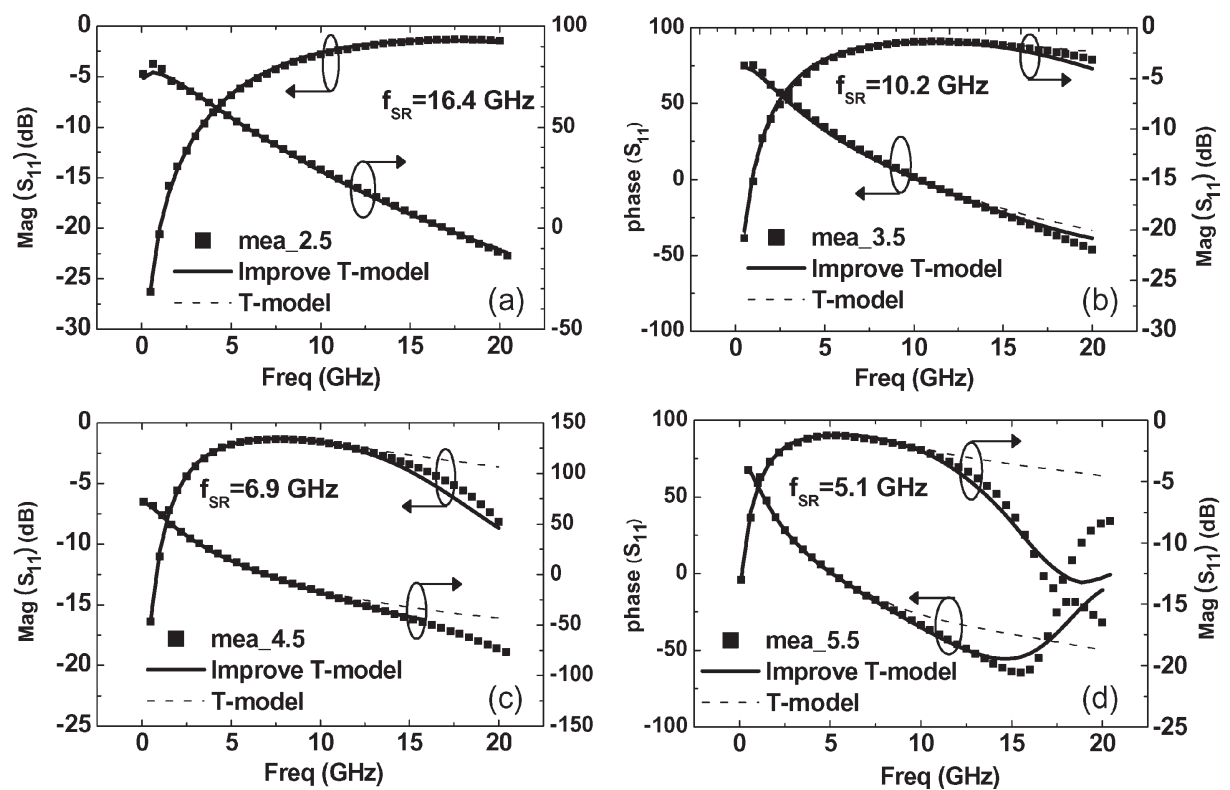

Fig. 5. Comparison between the improved T-model, original T-model, and measurement for $S_{11}$ (magnitude, phase) of inductors with various coil numbers. (a) $N=2.5$. (b) $N=3.5$. (c) $N=4.5$. (d) $N=5.5$. Improved T-model (solid lines). Original T-model (dash lines).

particularly in a higher frequency region. In addition, an even better agreement is obtained when compared with the EM simulation before a dedicated calibration. For $S_{11}$ shown in Fig. 5(a)-(d), it is reconfirmed that a good agreement with the measurement was achieved by the improved T-model in both magnitude and phase for all inductors through $20 \mathrm{GHz}$. Moreover, an improvement over the original T-model is demonstrated in a very high-frequency region. A more extensive verification has been performed by comparison of the three key parameters for spiral inductors, i.e., $L(\omega)\left(=\operatorname{Im}\left(\mathrm{Z}_{\text {in }}(\omega) / \omega\right)\right)$, $\operatorname{Re}\left(\mathrm{Z}_{\text {in }}(\omega)\right)$, and $Q(\omega)$. Herein, $Q(\omega)$ is the quality factor defined by $L(\omega) / \operatorname{Re}\left(\mathrm{Z}_{\text {in }}(\omega)\right)$. All three parameters are frequency dependent, which are critically related to the spiral conductor loss and Si substrate loss. Fig. 6(a)-(d) illustrates the excellent fit to the measured $L(\omega)$ and $\operatorname{Re}\left(\mathrm{Z}_{\mathrm{in}}(\omega)\right)$ by the improved T-model for all inductors operating up to $20 \mathrm{GHz}$. The transition from an inductive mode to a capacitive mode at $f>f_{\mathrm{SR}}$ is accurately reproduced. In addition, the model can exactly capture the full band feature of $\operatorname{Re}\left(\mathrm{Z}_{\mathrm{in}}(\omega)\right)$ even beyond the resonance, such as a dramatic increase prior to the resonance, a peak at resonance, and then a sharp fall-off after the peak. Eventually, $Q(\omega)$ is the most critical parameter governing RF IC performance such as power, gain, noise figure, and phase noise. Fig. 7 indicates the excellent match with the measured $Q(\omega)$ over a broadband of $20 \mathrm{GHz}$. The exact fit to the peak $Q$ and the capture of a full band behavior for all coil numbers suggest the advantage of the improved T-model compared to the existing $\pi$ model. 

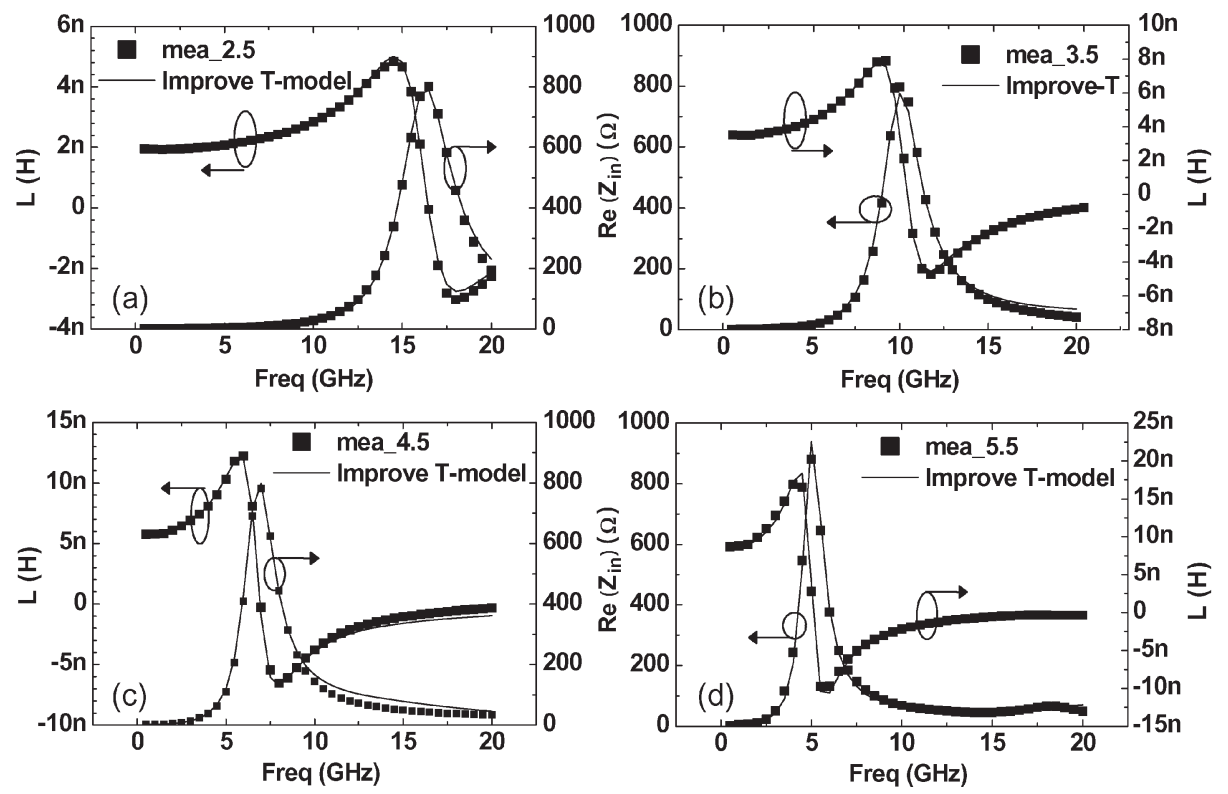

Fig. 6. Comparison between the improved T-model and measurement. $L(\omega)$ and $\operatorname{Re}\left(\mathrm{Z}_{\text {in }}(\omega)\right)$ for inductors with various coil numbers. (a) $N=2.5$. (b) $N=3.5$. (c) $N=4.5$. (d) $N=5.5$.

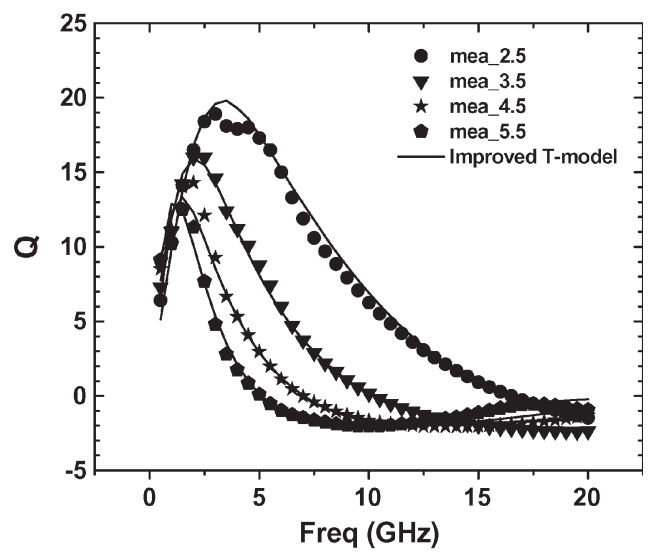

Fig. 7. Comparison between the improved T-model and measurement. $Q(\omega)$ for inductors with various coil numbers $N$ of $2.5,3.5,4.5$, and 5.5.

Note that the T-shape parallel $R L$ network adopted in the improved T-model can effectively improve the broadband accuracy, such as two-port $S$-parameters in very high frequencies beyond $f_{\mathrm{SR}}$ (Figs. 4 and 5), but has negligible effect on the quality factor $Q$ in frequencies below $f_{\mathrm{SR}}$. The resulting feature can be explained in part by the observation that the introduced normal component of substrate eddy currents cannot induce a normal magnetic field, and then has insignificant influence on the coil current distribution as well as the associated $Q$. On the other hand, the $R L$ elements introduced in the path that is in series with $C_{\mathrm{ox} 1} / C_{\mathrm{ox} 2}$ make the coupling between the spiral coils and the substrate underneath more complicated than a pure capacitive coupling and, then, alter the behavior in very high frequencies such as above $f_{\mathrm{SR}}$.

For inductors in the second category of varying metal trace widths, the broadband accuracy was verified following previous procedure performed for the first one. First, Fig. 8(a)-(d) presents the comparison between the model and measurement for the magnitude and phase of $S_{21}$. A good match is demonstrated on phase $\left(S_{21}\right)$ for all widths
$(W=3,9,15,30 \mu \mathrm{m})$ over a broadband of up to $20 \mathrm{GHz}$. With regard to $\operatorname{mag}\left(S_{21}\right)$, a somewhat larger deviation that was revealed at higher frequency for the largest inductor with $W=30 \mu \mathrm{m}$ is considered due to the increased proximity effect. Through our early study, it can be improved by taking an inductive element in series with $R_{P}$ to emulate the proximityeffect-induced eddy current in metal coils. More thorough investigation will be continued as a future work. Afterward, Fig. 9(a)-(d) indicates the excellent agreement between the model and measurement in $\operatorname{mag}\left(S_{11}\right)$ and phase $\left(S_{11}\right)$ for all widths and over a broadband of $20 \mathrm{GHz}$. In addition, Fig. 10(a) and (b) demonstrates the precise match with measured $L(\omega)$ and $\operatorname{Re}\left(\mathrm{Z}_{\text {in }}(\omega)\right)$ by the inductor model for all geometries.

Note that the inductor with the narrowest width, i.e., $W=3 \mu \mathrm{m}$, reveals the largest inductance at lower frequency, whereas the inductor with the widest width, i.e., $W=30 \mu \mathrm{m}$, takes the place of the largest inductance at higher frequency. The remarkable increase of $L$ and $\operatorname{Re}\left(\mathrm{Z}_{\text {in }}\right)$ for the inductor with the widest metal $(W=30 \mu \mathrm{m})$ at higher frequency suggests the larger capacitive coupling effect originated from larger metal coverage area. Finally, Fig. 11(a)-(d) indicates that the improved T-model can predict $Q(\omega)$ with good accuracy over a full range of $20 \mathrm{GHz}$ for all inductors with different metal trace widths. Following the previous observation of metal width effect on $L(\omega)$ and $\operatorname{Re}\left(\mathrm{Z}_{\text {in }}(\omega)\right)$, the wider metal width helps raise the maximum $Q$ but will have a disadvantage of lower self-resonance frequency $f_{\mathrm{SR}}$ and a narrow bandwidth due to increased capacitive coupling effect.

\section{B. Model Scalability for Various Geometries}

Following the proven broadband accuracy, another important feature to be verified and justified is the scalability with respect to the inductor geometries for all model parameters. At first, the evaluation was performed on the first category of inductors with various coil numbers $(N=2.5,3.5,4.5,5.5)$ and a 

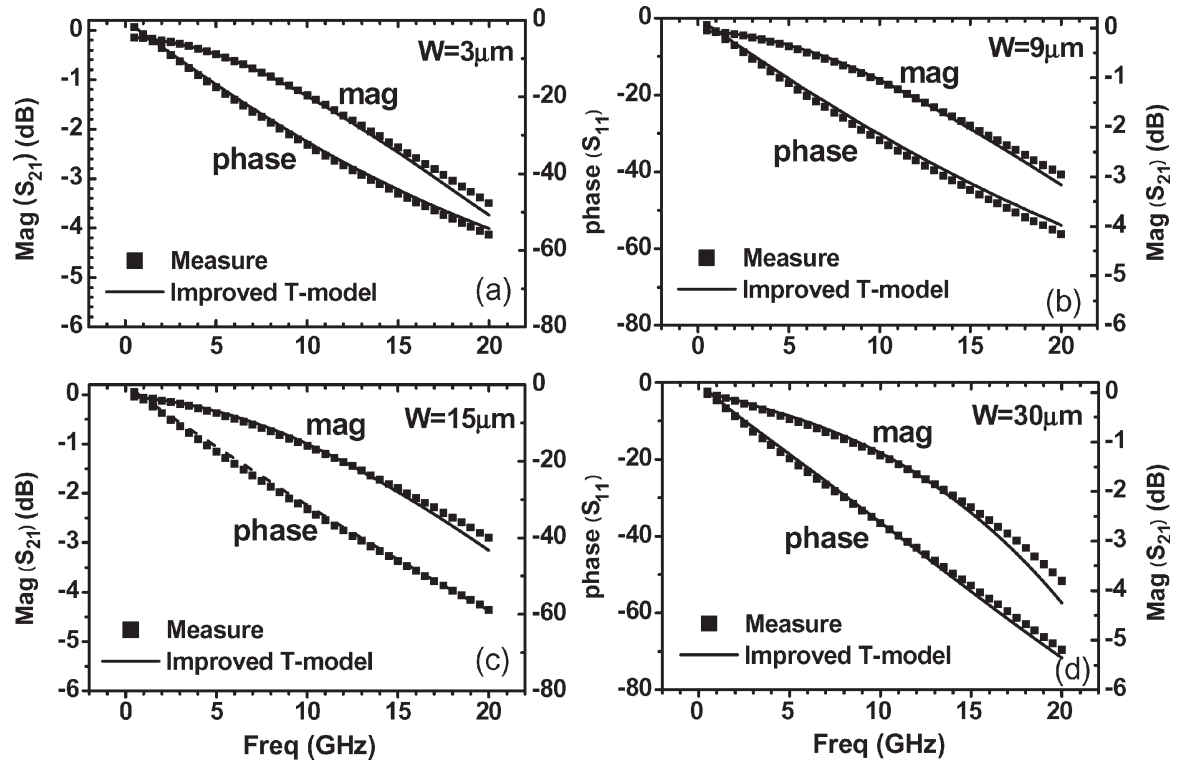

Fig. 8. Comparison between the improved T-model and measurement. $S_{21}$ (magnitude, phase) for inductors with various metal trace widths. (a) $W=3 \mu$ m. (b) $W=9 \mu \mathrm{m}$. (c) $W=15 \mu \mathrm{m}$. (d) $W=30 \mu \mathrm{m}$.
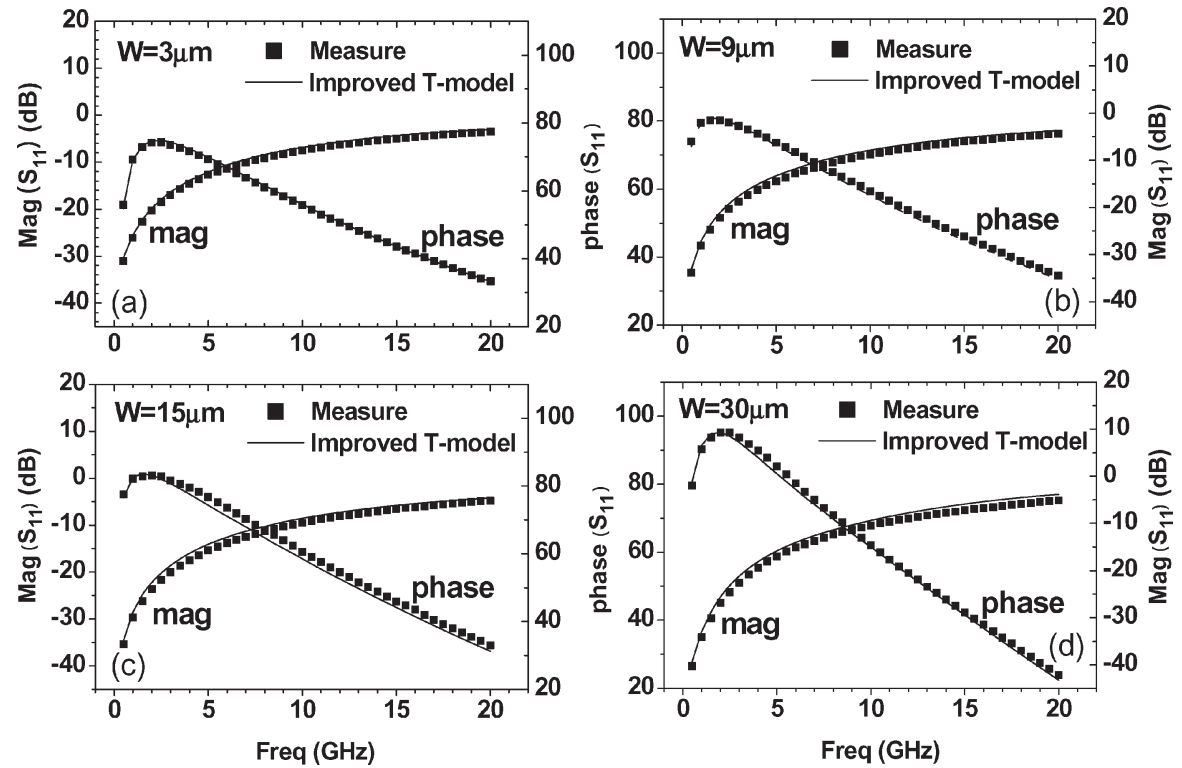

Fig. 9. Comparison between the improved T-model and measurement. $S_{11}$ (magnitude, phase) for inductors with various metal trace widths. (a) $W=3 \mu$ m. (b) $W=9 \mu \mathrm{m}$. (c) $W=15 \mu \mathrm{m}$. (d) $W=30 \mu \mathrm{m}$.
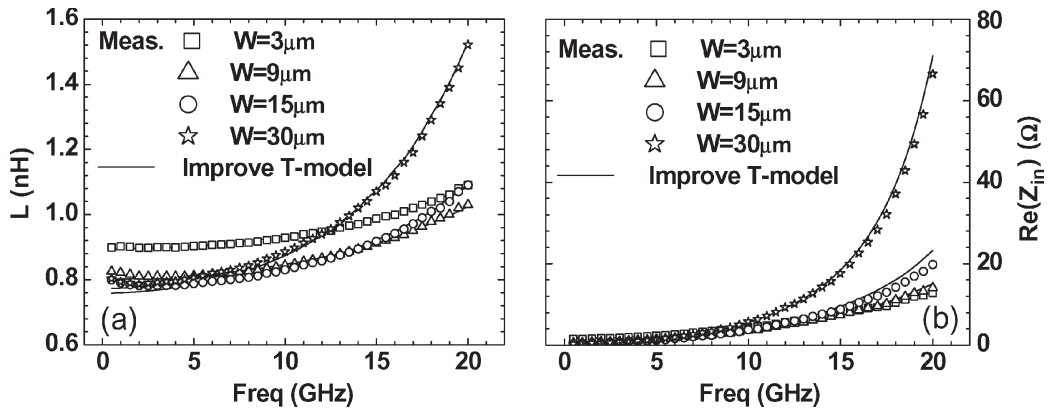

Fig. 10. Comparison between the improved T-model and measurement. (a) $L(\omega)$ and (b) $\operatorname{Re}\left(\mathrm{Z}_{\mathrm{in}}(\omega)\right)$ for inductors with various metal trace widths $W$ of 3,9 , 15 , and $30 \mu \mathrm{m}$. 

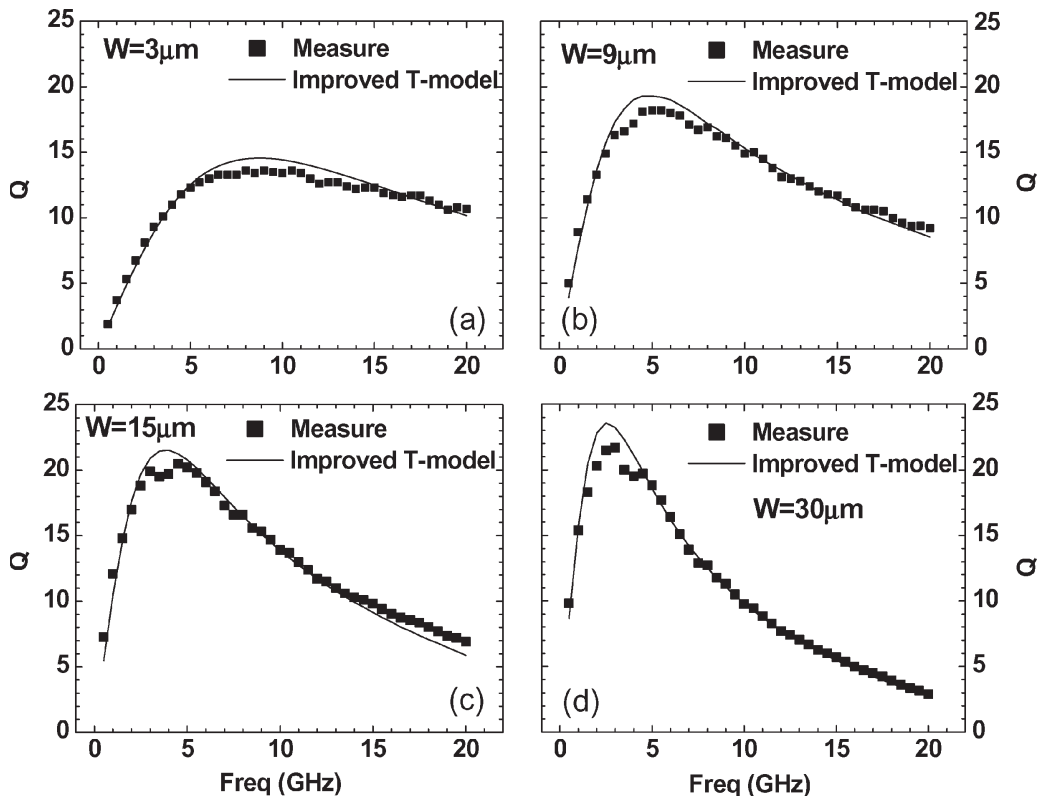

Fig. 11. Comparison between the improved T-model and measurement. $Q(\omega)$ for inductors with various metal trace widths. (a) $W=3 \mu \mathrm{m}$. (b) $W=9 \mu \mathrm{m}$. (c) $W=15 \mu \mathrm{m}$. (d) $W=30 \mu \mathrm{m}$.
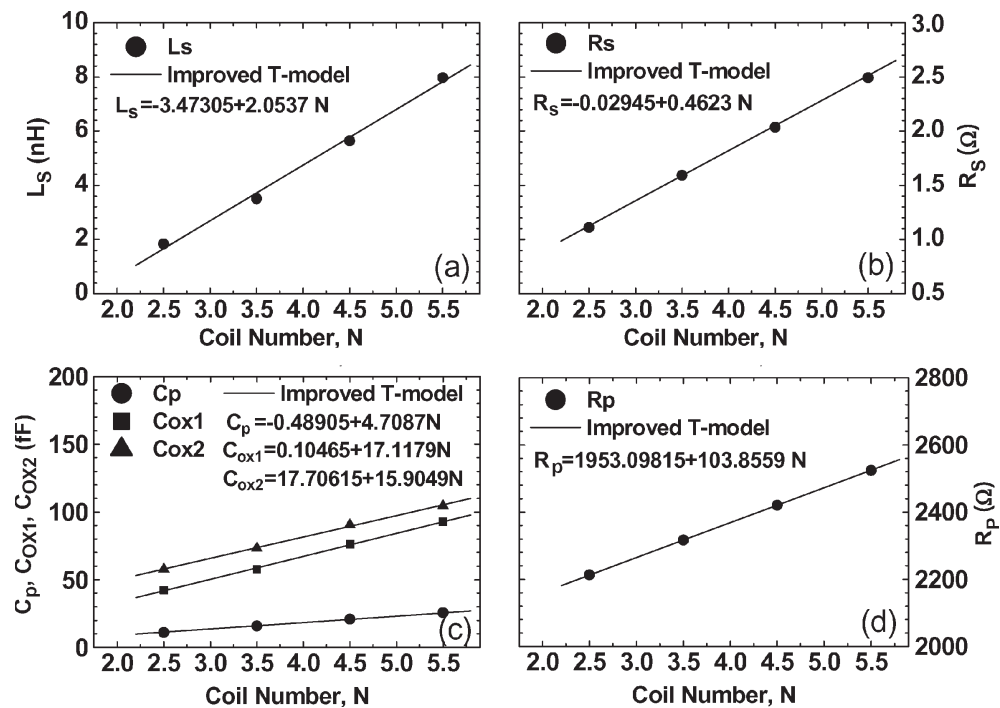

Fig. 12. Improved T-model parameters versus inductor coil numbers $(N=2.5,3.35,4.5,5.5)$. (a) $L_{S}$. (b) $R_{S}$. (c) $C_{P}$ and $C_{\mathrm{ox} 1,2}$. (d) $R_{P}$.

fixed width $(W=15 \mu \mathrm{m})$. Fig. 12(a)-(d) presents a good match with a linear function of coil numbers for each model parameter in the spiral coil's $R L C$ network, i.e., $L_{S}, R_{S}, R_{P}$, $C_{P}$, and $C_{\text {ox } 1,2}$. Fig. 13(a)-(d) indicates the excellent fit by a linear function for the substrate-network-involved model parameters, such as $L_{\mathrm{sub}}, L_{\mathrm{sub} 1,2}, C_{\mathrm{sub}}, 1 / R_{\mathrm{sub}}, R_{\text {loss }}$, and $R_{\text {loss1,2 }}$. All $R L C$ elements in the model with the exception of $R_{\text {sub }}$ increase with increasing coil numbers. The smaller $R_{\text {sub }}$ corresponding to larger coil number accounts for the worse substrate loss effect.

Subsequently, the verification was performed on the second category of inductors with varying metal widths $(W=3,9,15,30 \mu \mathrm{m})$ and a fixed coil number $(N=1.5)$. Fig. 14(a)-(d) illustrates a good fit with a parabolic function of width for each model parameter in the spiral coil's $R L C$ network, i.e., $L_{S}, R_{S}, R_{P}$, and $C_{P}$. Among these four elements in the coil network, both inductive and resistive elements, i.e., $L_{S}, R_{S}$, and $R_{P}$, decrease with increasing width, whereas the capacitive element $C_{P}$ goes the opposite trend. The results are consistent with the prediction from the physical layout that the larger width leads to smaller square number at a fixed coil number, i.e., metal length and then the smaller resistance $R_{S}$, while the larger metal overlap area will contribute to larger capacitance $C_{P}$. Furthermore, the smaller $R_{P}$ associated with larger $W$ accounts for the worse substrate return loss. Note that the variation with width reveals a trend of saturation for larger width beyond $30 \mu \mathrm{m}$. Moreover, as shown in Fig. 15(a)-(d), all the model parameters of the substrate $R L C$ network, i.e., $C_{\text {sub }}, 1 / R_{\text {sub }}, L_{\text {sub }}$, and $R_{\text {loss }}$, perfectly follow a parabolic function of width. Note that $C_{\text {sub }}$ and $L_{\text {sub }}$ increase with increasing width, whereas $R_{\text {sub }}$ and $R_{\text {loss }}$ reveal the opposite trend. The larger $C_{\text {sub }}$ and 

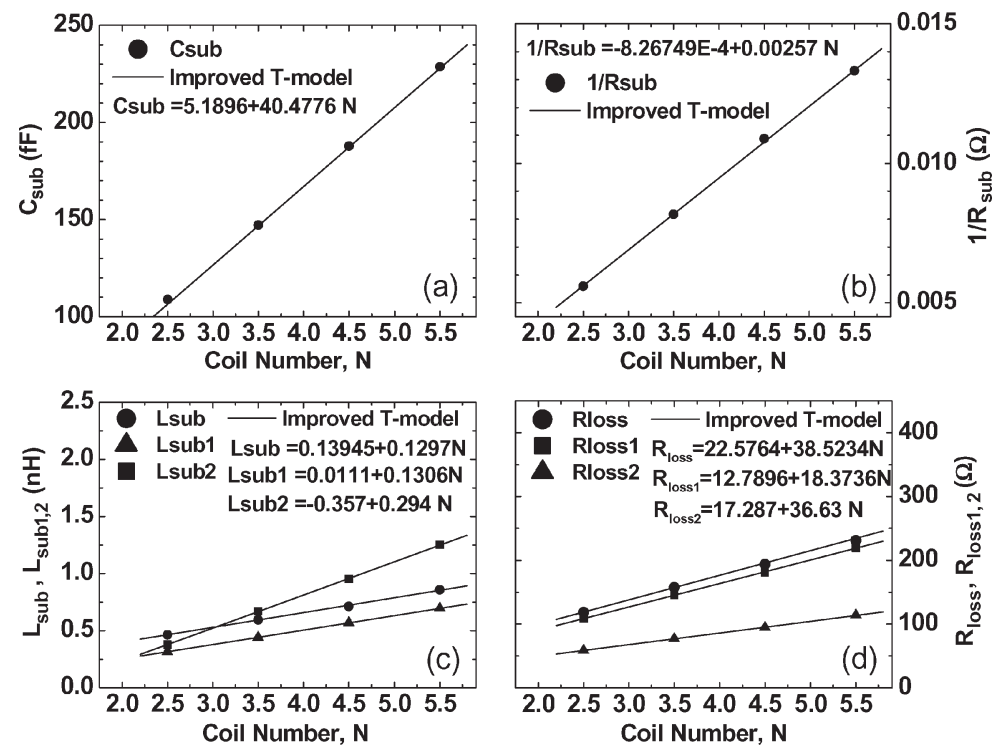

Fig. 13. Improved T-model parameters versus inductor coil numbers $(N=2.5,3.35,4.5,5.5)$. (a) $C_{\mathrm{sub}}$. (b) $1 / R_{\mathrm{sub}}$. (c) $L_{\mathrm{sub}}$ and $L_{\mathrm{sub} 1,2}$. (d) $R_{\text {loss }}$ and $R_{\text {loss } 1,2}$.
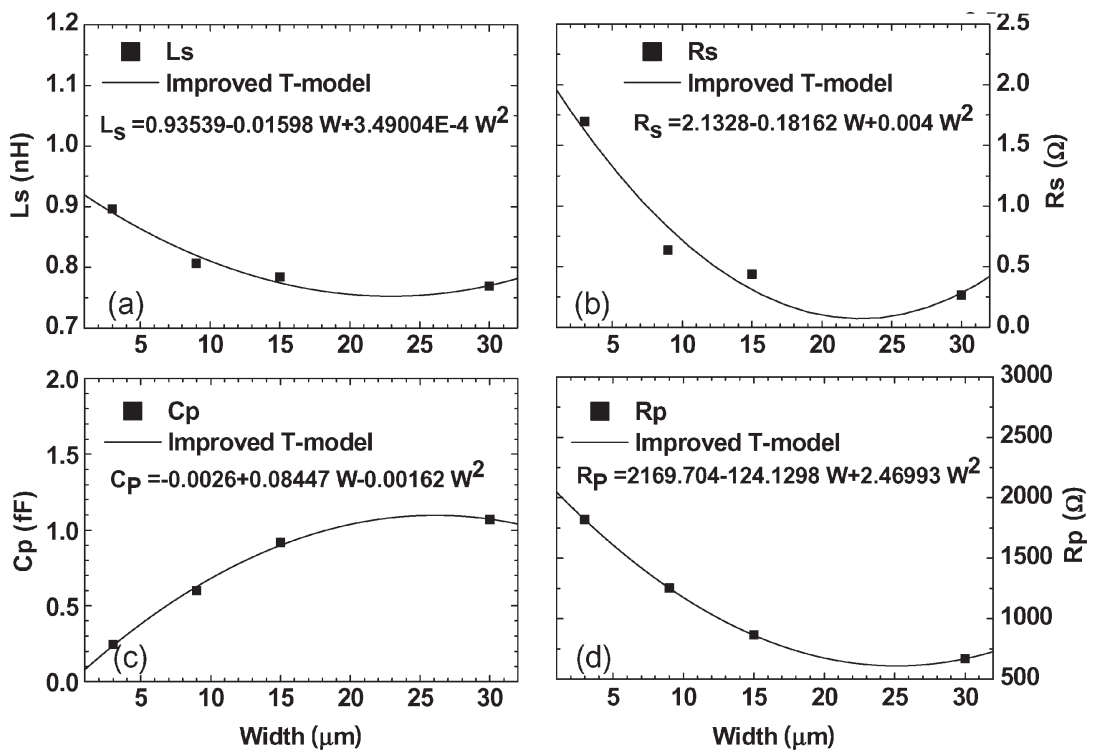

Fig. 14. Improved T-model parameters versus metal trace widths $(W=3,9,15,30 \mu \mathrm{m})$. (a) $L_{S}$. (b) $R_{S}$. (c) $C_{P}$. (d) $R_{P}$.

$L_{\text {sub }}$ contribute the larger capacitive coupling and substrate eddy current effects that are caused by larger metal coverage area due to wider metal coils. The smaller $R_{\text {sub }}$ and $R_{\text {loss }}$ address the worse substrate loss corresponding to larger inductors of wider metal area. Finally, $C_{\mathrm{ox} 1}$ and $C_{\mathrm{ox} 2}$ that are shown in Fig. 16 indicate the larger capacitances associated with a wider metal, which is consistent with the physical layout.

The model scalability proven over a wide range of geometries, as demonstrated, suggests that this T-model is useful for prelayout simulation and can facilitate an inductor optimization.

\section{Operation Modes of VARYing SUbSTRATE RESISTIVITIES}

EM simulation using Agilent ADS momentum with an extensive calibration on $0.13-\mu \mathrm{m} \quad \mathrm{Cu}$ BEOL technology parameters was conducted to explore the broadband characteristics of inductors under varying substrate resistivities $\rho_{\mathrm{Si}}$. The first goal is to verify the three operation modes predicted by wave propagation analysis that was carried out for microstrip lines in the $\mathrm{Si}-\mathrm{SiO}_{2}$ system [7]. Afterward, the improved T-model parameters will be extracted to verify if this model is applicable for an on-Si-chip inductor design with the freedom of varying substrate resistivities. The ultimate goal is to realize a scalable model incorporating the substrate resistivity effect to accurately simulate on-Si-chip inductor performance and fit for Si-based RF circuit design.

\section{A. EM Simulation for Substrate Resistivity Effect on Inductors}

Fig. 17 indicates a good match between ADS momentum, measurement, and T-model in terms of $S_{11}, S_{21}, L(\omega)$, $\operatorname{Re}\left(\mathrm{Z}_{\text {in }}(\omega)\right)$, and $Q(\omega)$ for inductors on a standard Si substrate 

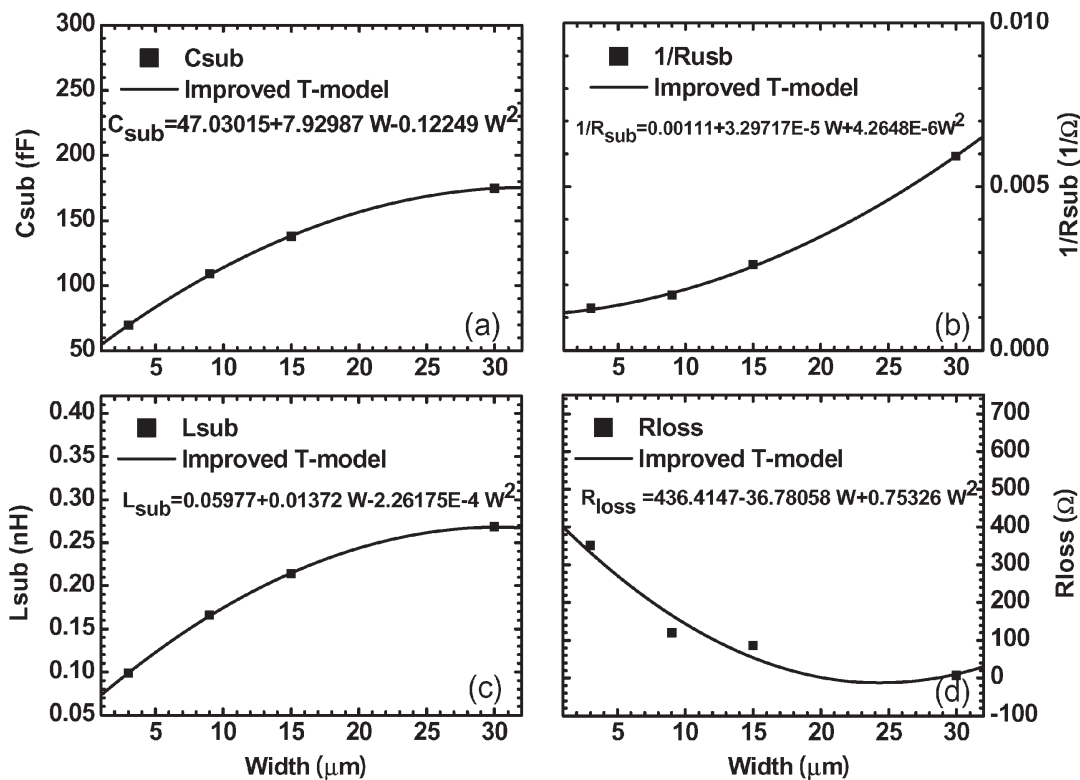

Fig. 15. Improved T-model parameters versus metal trace widths $(W=3,9,15,30 \mu \mathrm{m})$. (a) $C_{\mathrm{sub}}$. (b) $1 / R_{\mathrm{sub}}$. (c) $L_{\mathrm{sub}}$. (d) $R_{\text {loss }}$.
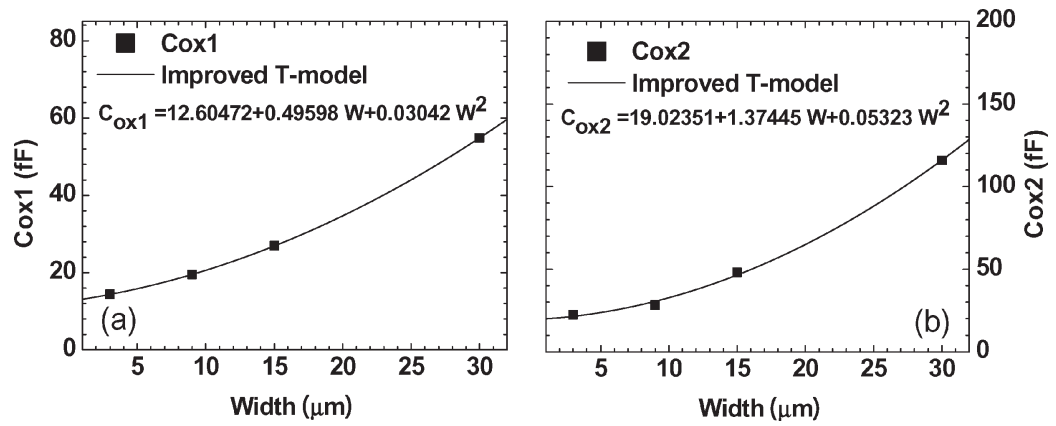

Fig. 16. Improved T-model parameters versus metal trace widths $(W=3,9,15,30 \mu \mathrm{m})$. (a) $C_{\mathrm{ox} 1}$. (b) $C_{\mathrm{ox} 2}$.
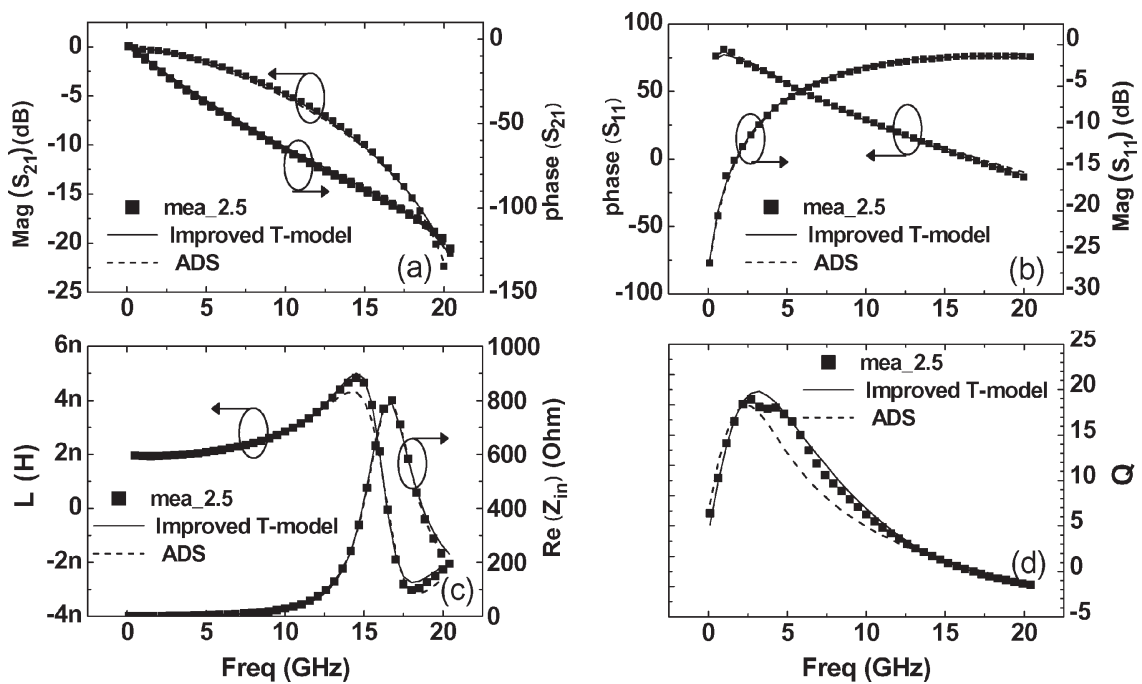

Fig. 17. Comparison between ADS momentum simulation, measurement, and improved T-model for on-chip inductor. (a) $S_{11}$ (magnitude, phase). (b) $S_{21}$ (magnitude, phase). (c) $L(\omega)$ and $\operatorname{Re}\left(\mathrm{Z}_{\text {in }}(\omega)\right)$. (d) $Q(\omega) \cdot \rho_{\mathrm{Si}}=10 \Omega \cdot \mathrm{cm}$ defined for simulation.

of $\rho_{\mathrm{Si}}=10 \Omega \cdot \mathrm{cm}$. Using the calibrated ADS momentum, three operation modes such as TEM mode, slow-wave mode, and eddy current mode [7] corresponding to a wide range of $\rho_{\mathrm{Si}}(0.05-1 \mathrm{k} \Omega \cdot \mathrm{cm})$ are reproduced. Fig. $18(\mathrm{a})-(\mathrm{d})$ presents four key parameters, i.e., $Q_{m}, f_{m}, f_{\mathrm{Lmax}}$, and $f_{\mathrm{SR}}$, as a function of $\rho_{\mathrm{Si}} . Q_{m}$ is the maximum $Q$, and $f_{m}$ is the frequency responsible for $Q_{m} . f_{\mathrm{Lmax}}$ is the frequency corresponding to the maximum $L$. An interesting result is identified in the 

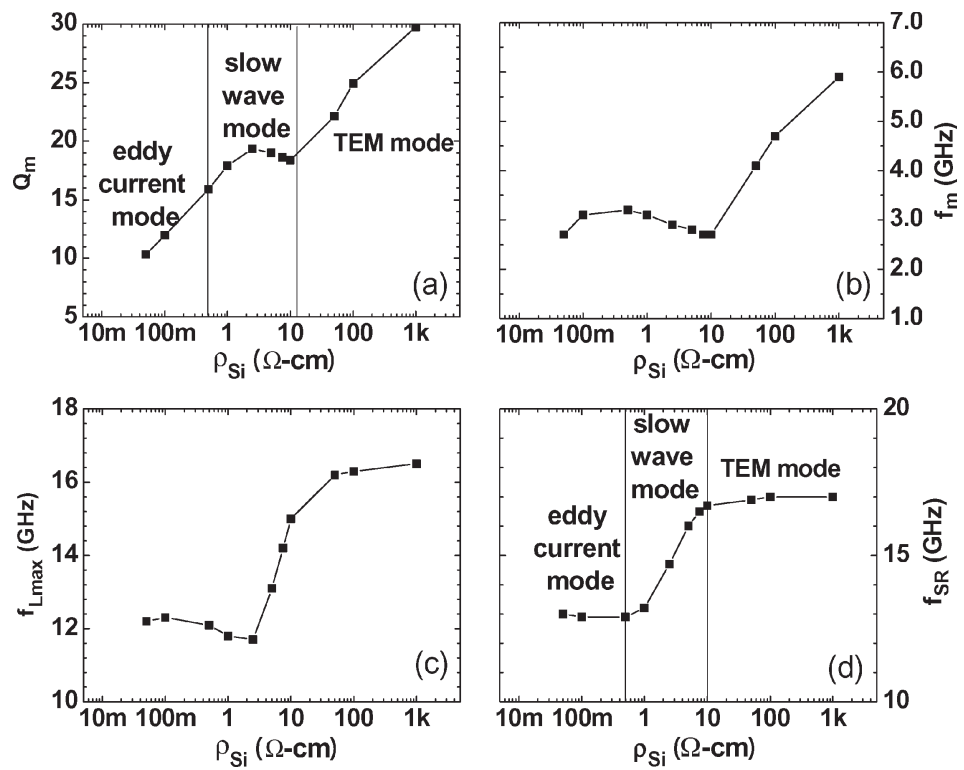

Fig. 18. ADS momentum simulation for prediction of (a) $Q_{m}$, (b) $f_{m}$, (c) $f_{\mathrm{Lmax}}$, and (d) $f_{\mathrm{SR}}$ under varying $\rho_{\mathrm{Si}}(0.01-1 \mathrm{k} \Omega \cdot \mathrm{cm})$.
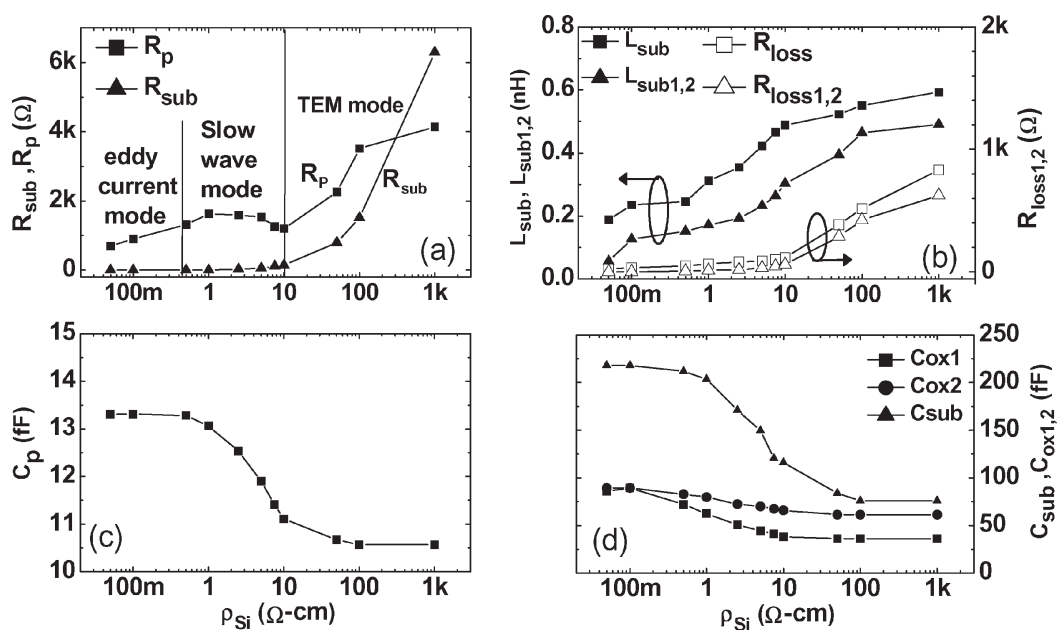

Fig. 19. Improved T-model parameters extracted to fit the inductors' characteristics under varying $\rho_{\mathrm{Si}}$. (a) $R_{\mathrm{sub}}$ and $R_{P}$. (b) $L_{\mathrm{sub}}, L_{\mathrm{sub} 1,2}, R_{\mathrm{loss}}$, and $R_{\text {loss } 1,2}$ (c) $C_{P}$. (d) $C_{\mathrm{ox} 1,2}$ and $C_{\mathrm{sub}}$.

region of $\rho_{\mathrm{Si}}=0.5-10 \Omega \cdot \mathrm{cm}$, where $f_{\mathrm{SR}}$ drops monotonically with decreasing $\rho_{\mathrm{Si}}$ while $Q_{m}$ reveals a hump due to an initial increase and then a fall-off with further reduction of $\rho_{\mathrm{Si}}$. The fall-off of $f_{\mathrm{SR}}$ and the increase of $Q_{m}$ suggest that the spiral coil is approaching the resonator mode, i.e., the slow-wave mode. For a high resistivity region of $\rho_{\mathrm{Si}}>$ $10 \Omega \cdot \mathrm{cm}, f_{\mathrm{SR}}$ saturates at a maximum while $Q_{m}$ increases continuously with $\rho_{\mathrm{Si}}$. This region is the so-called TEM mode or inductor mode, which favors the inductor operation with high $Q$ attributed to suppressed resonance in a substrate of dielectric property. Note that the saturation of $f_{\mathrm{SR}}$ under further increasing substrate resistivities beyond the standard $\mathrm{Si}$, i.e., $\rho_{\mathrm{Si}}>10 \Omega \cdot \mathrm{cm}$, can be supported by the experimental results reported in the 2003 IEEE International Electron Devices Meeting [8], in which ultrahigh resistivity substrates with $\rho_{\mathrm{Si}}>10^{4}-10^{5} \Omega \cdot \mathrm{cm}$ were achieved through proton bombardment, which effectively raised $Q$ by around $100 \%$ but retained an almost negligible change in $f_{\mathrm{SR}}$. With regard to the very low resistivity region of $\rho_{\mathrm{Si}}<0.5 \Omega \cdot \mathrm{cm}$,
$f_{\mathrm{SR}}$ saturates at a minimum and $Q_{m}$ drops drastically. The spiral coil is driven into the eddy current mode or the skin effect mode, whe re $\rho_{\mathrm{Si}}$ is so small that the skin depth is thinner than the substrate thickness and becomes the limiting factor.

\section{B. Improved T-Model Parameters Under Varying Substrate Resistivities}

In the following, the improved T-model parameters are extracted from the simulated $S$-parameters under various $\rho_{\mathrm{Si}}$ to verify if the model parameters can reflect the physical properties responsible for the three modes of operation. Fig. 19(a) and (b) indicates how the resistive elements $\left(R_{P}, R_{\mathrm{sub}}, R_{\text {loss }}, R_{\text {loss1 } 1,2}\right)$ and inductive elements $\left(L_{\mathrm{sub}}, L_{\mathrm{sub} 1,2}\right)$ vary with varying $\rho_{\mathrm{Si}}$. Quite interestingly, $R_{P}$ exactly follows the same trend as that of $Q_{m}$ versus $\rho_{\mathrm{Si}}$ with a hump in slow-wave mode, while the others show a monotonic increase with $\rho_{\mathrm{Si}}$ in slow-wave and TEM modes and near saturation at a minimum in eddy current mode. With regard 

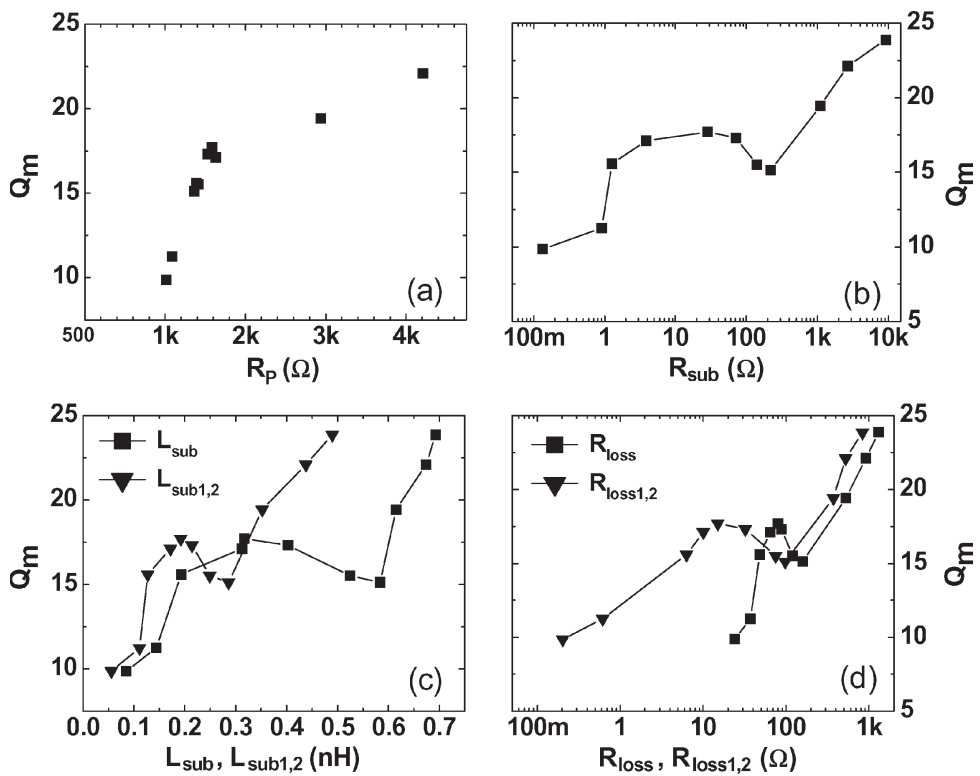

Fig. 20. Maximum quality factor $Q_{m}$ versus resistance parameters in improved T-model. (a) $R_{P}$. (b) $R_{\text {sub }}$ (c) $L_{\text {sub }}$ and $L_{\text {sub1,2 }}$ (d) $R_{\text {loss }}$ and $R_{\text {loss } 1,2}$.
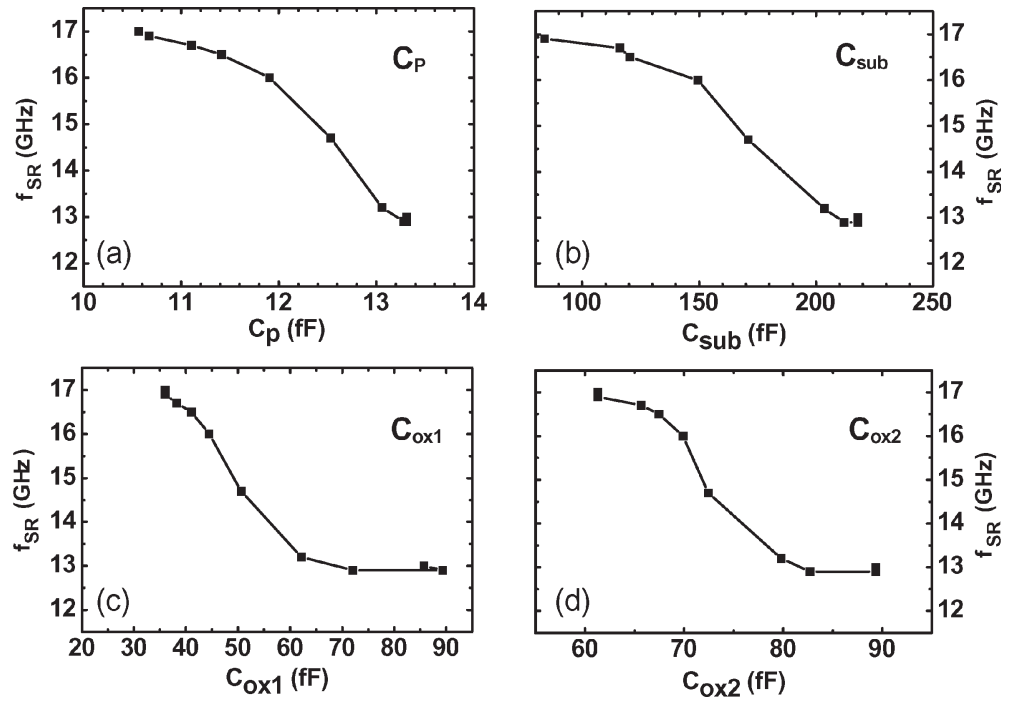

Fig. 21. Self-resonance frequency $f_{\mathrm{SR}}$ versus capacitance parameters in improved T-model. (a) $C_{P}$. (b) $C_{\mathrm{sub}}$ (c) $C_{\mathrm{ox} 1}$. (d) $C_{\mathrm{Ox} 2}$.

to the capacitive elements $\left(C_{P}, C_{\mathrm{sub}}, C_{\mathrm{ox} 1}, C_{\mathrm{ox} 2}\right)$ in Fig. 19(c) and (d), all four capacitances demonstrate a monotonic increase with a reduction of $\rho_{\mathrm{Si}}$ in slow-wave mode, saturation at a minimum in TEM mode, while different behaviors in eddy current mode. The larger capacitances that are associated with lower $\rho_{\mathrm{Si}}$ in the slow-wave mode only play a major role in the drastic fall-off of $f_{\mathrm{SR}}$ with decreasing $\rho_{\mathrm{Si}}$. With regard to the $R L C$ model parameter effect in determining $Q_{m}$ and $f_{\mathrm{SR}}$ over a wide range of $\rho_{\mathrm{Si}}$, Fig. 20 reveals a monotonic increase of $Q_{m}$ due to an increase of $R_{P}$, while a hump appears for other resistive or inductive elements, such as $R_{\text {sub }}, R_{\text {loss }}$, and $L_{\text {sub }}$, corresponding to the region of slow-wave mode. Referring to our previous work [6], $R_{P}$ reveals a significant effect on $Q$ over a broad bandwidth from frequency below $f_{m}\left(Q_{m}\right)$ to that well above $f_{m}\left(Q_{m}\right)$. The interesting results support our proposed mechanism that $R_{P}$, which is a new element introduced in our T-model, is the key parameter to simulate an excess energy loss in the substrate and metal coils. The mutual interaction between the metal coils and the substrate underneath via the generation of magnetic fluxes and eddy currents is referred to as the "substrate return path," which is discussed in Section III. The results achieved through this study suggest that $R_{P}$ is the most critical parameter to explicitly guide the substrate engineering for on-Si-chip inductors attaining the maximum $Q_{m}$. Fig. 21 presents the capacitances' $\left(C_{P}, C_{\mathrm{sub}}, C_{\mathrm{ox} 1}, C_{\mathrm{ox} 2}\right)$ effect on $f_{\mathrm{SR}}$, where the monotonic increase of $f_{\mathrm{SR}}$ with decreasing capacitances is demonstrated for all four capacitances.

\section{CONCLUSION}

A broadband and scalable model has been developed to accurately simulate on-chip spiral inductors operating up to $20 \mathrm{GHz}$. The broadband accuracy has been proven by a good match with the measured $S$-parameters, $L(\omega), \operatorname{Re}\left(Z_{\text {in }}(\omega)\right)$, and 
$Q(\omega)$ over a wideband up to $20 \mathrm{GHz}$. The scalability has been justified by a good fit with a linear function of coil numbers or a parabolic function of metal trace widths for all model parameters in the spiral inductors with varying coil numbers or metal widths, respectively. Noteworthily, the physics-based model parameters enable this model to be applicable in three operation modes under varying substrate resistivities. This broadband and scalable inductor model can facilitate an optimum design of on-Si-chip inductors and improve RF circuit simulation accuracy, which are the requirements in a broadband design.

\section{ACKNOWLEDGMENT}

The authors would like to thank the Chip Implementation Center and RF Laboratory in the National Device Laboratory for providing the high-frequency simulation and device measurement environment.

\section{REFERENCES}

[1] C. P. Yue and S. S. Wong, "Physical modeling of spiral inductors on silicon," IEEE Trans. Electron Devices, vol. 47, no. 3, pp. 560-568, Mar. 2000.

[2] M. Park, S. Lee, C. S. Kim, H. K. Yu, and K. S. Nam, "The detailed analysis of high $Q$ CMOS-compatible microwave spiral inductors in silicon technology," IEEE Trans. Electron Devices, vol. 45, no. 9, pp. 1953-1959, Sep. 1998.

[3] Y. Cao, R. A. Grove, X. Huang, N. D. Zamdmer, J.-O. Plouchart, R. A. Wachnik, T.-J. King, and C. Hu, "Frequency-independent equivalentcircuit model for on-chip spiral inductors," IEEE J. Solid-State Circuits, vol. 38, no. 3, pp. 419-426, Mar. 2003.

[4] M. Fujishima and J. Kino, "Accurate subcircuit model of an on-chip inductor with a new substrate network," in VLSI Symp. Tech. Dig., 2004, pp. 376-379.

[5] J. Gil and H. Shin, "A simple wide-band on-chip inductor model for siliconbased RF ICs," IEEE Trans. Microw. Theory Tech., vol. 51, no. 9, pp. $2023-$ 2028, Sep. 2003

[6] J.-C. Guo and T.-Y. Tan, "A broadband and scalable model for on-chip inductors incorporating substrate and conductor loss effects," IEEE Trans. Electron Devices, vol. 53, no. 3, pp. 413-421, Mar. 2006.

[7] H. Hasegawa, M. Furukawa, and H. Yanai, "Properties of microstrip line on $\mathrm{Si}-\mathrm{SiO}_{2}$ system," IEEE Trans. Microw. Theory Tech., vol. MTT-19, no. 11 , pp. 869-881, Nov. 1971.

[8] D. D. Tang, W. C. Lin, L. S. Lai, C. H. Wang, L. P. Lee, H. M. Hsu, C. M. Wu, C. W. Chang, W. Y. Lien, C. P. Chao, C. Y. Lee, G. J. Chern, J. C. Guo, C. S. Chang, Y. C. Sun, D. S. Du, K. C. Lan, and L. F. Lin, "The integration of proton bombardment process into the manufacturing of mixed-signal/RF chips," in IEDM Tech. Dig., Dec. 2003, pp. 673-676.

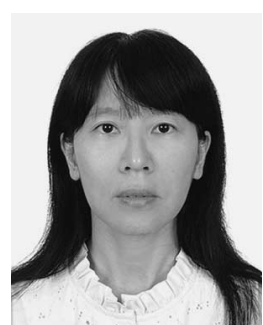

Jyh-Chyurn Guo (M'06-SM'07) received the B.S.E.E. and M.S.E.E. degrees from the National Tsing-Hua University, Hsinchu, Taiwan, R.O.C., in 1982 and 1984, respectively, and the Ph.D. degree in electronics engineering from the National Chiao Tung University (NCTU), Hsinchu, in 1994.

For more than 19 years, she was with the semiconductor industry, where her major focus was on device design and VLSI technology development. In 1984 , she joined the ERSO/ITRI, where she had been engaged in semiconductor integrated circuit technologies with broad scope that covers high voltage, high power, submicrometer project, high-speed SRAM technologies, etc. From 1994 to 1998, she was with Macronix International Corporation, where she was engaged in the highdensity and low-power Flash memory technology development. In 1998, she joined Vanguard International Semiconductor Corporation, where she assumed the responsibility of the Device Department Manager for advanced DRAM device technology development. In 2000, she joined Taiwan Semiconductor Manufacturing Company, where she served as a Program Manager in charge of the 100-nm logic CMOS FEOL, high-performance analog, and RF CMOS technology development. Since 2003, she has been with the Department of Electronics Engineering, NCTU, as an Associate Professor. She has authored or coauthored around 50 technical papers and is the holder of 19 U.S. patents in her professional field. Her current research interests cover RF CMOS and highperformance analog device design and modeling, novel nonvolatile memory technology, and device integration technologies for SOC.

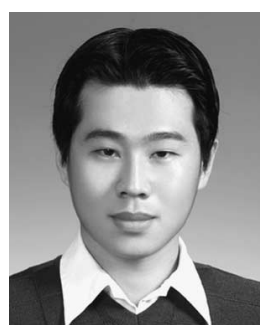

Teng-Yang Tan was born in Taichung, Taiwan, R.O.C., in 1981. He received the M.S. degree in electronics engineering from the National Chiao Tung University (NCTU), Hsinchu, Taiwan, in 2006.

$\mathrm{He}$ is currently with the Department of Electronics Engineering, NCTU. His current research interests focus on RF passive device modeling and characterization. 